\title{
Mediciones de adiposidad intraabdominal por ultrasonido $y$ factores asociados con riesgo cardiovascular en niños obesos
}

\author{
MARCELA REYES ${ }^{1}$, ANÍBAL ESPINOZA ${ }^{2}$, M. JESÚS REBOLLO ${ }^{2}$, \\ FRANCISCO MORAGA ${ }^{2}$, VERÓNICA MERICQ ${ }^{4}$, CARLOS CASTILLO-DURÁN $^{3}$
}

Instituto de Nutrición y Tecnología de los Alimentos (INTA), Universidad de Chile ${ }^{2}$ Hospital San Borja-Arriarán Santiago de Chile. ${ }^{3}$ Departamento de Pediatría. ${ }^{4}$ Instituto de Investigaciones Materno Infantil (IDIMI), Facultad de Medicina, Universidad de Chile, Santiago de Chile.

Recibido el 8 de abril de 2009 aceptado el 7 de enero de 2010.

Correspondencia a:

Marcela Reyes Jedlicki INTA, Universidad de Chile El Líbano 5524, Casilla 138-11, Santiago, Chile Teléfono 56-2-9781503

Fax: $56-2-2214030$ E-mail:mreyes@inta.cl

\section{Ultrasound measurements of intra-abdominal adiposity and factors associated with cardiovascular risk in obese children}

Background: Cardiovascular risk factors are commonly present in obese children. Aim: To evaluate the association among radiological measurements of intra-abdominal adipose tissue, and cardiovascular risk factors, in prepuberal obese children. Patients and Methods: We evaluated 30 obese (body mass index $\geq p 95$ ) children aged 6 to 12 years (15 males). Anthropometry and blood pressure were measured. Subcutaneous and intra-abdominal fat thickness and fat area were measured by ultrasound (US) and computed tomography. Serum insulin, glucose and lipid profile were measured in a fasting blood sample. Homeostasis model assessment (HOMA) was calculated as an index of insulin resistance. Results: There was a significant correlation between US intra-abdominal fat thickness and HOMA $(r=0.47$, $p<0.01)$, serum triglycerides $(r=0.46, p<0.05)$ and with positive criteria for metabolic syndrome $(r=0.66, p<0.01)$. A receiver operating curve $(R O C)$ analysis showed that, above a cut-off of $45 \mathrm{~mm}$ for intra-abdominal fat thickness, US was able to identify insulin resistance with a sensibility and specificity of 79 and $69 \%$ respectively and metabolic syndrome with sensibility and specificity of 100 and $67 \%$ respectively. US and computed tomography measurements for intra-abdominal fat thickness were significantly correlated $(r=0.62, p<0.01)$. Conclusions: US measurements of intra-abdominal fat thickness identify obesity-associated damage in childhood. Age-specific measurements of intra-abdominal adipose tissue may improve the detection power of this approach.

(Rev Med Chile 2010; 138: 152-159).

Key words: Adiposity; Child; Obesity, abdominal; Ultrasonography.
L a obesidad ha sido catalogada como una epidemia mundial, de aparición cada vez más temprana y severa. Los factores de riesgo cardiovascular asociados a ella ya están presentes en la edad pediátrica, condicionando una generación actual de niños que padecería de mayor morbilidad y tendría un menor tiempo de vida que el de sus padres ${ }^{1}$.

El balance energético positivo mantenido en el tiempo determina la formación y almacenamiento de triglicéridos, condicionando un aumento de la masa del tejido adiposo, lo que se conoce como obesidad. Este fenómeno está asociado a la aparición de factores de riesgo cardiovascular como son resistencia insulínica, diabetes mellitus tipo 2, hipertensión arterial y dislipidemia, entre otros. Hay múltiples antecedentes que asocian el tamaño del depósito graso intraabdominal con la presencia de 
las comorbilidades de la obesidad, en las diferentes etapas de la vida, aun cuando no se sabe si esta asociación es simple o causal ${ }^{2-5}$. La medición de la circunferencia abdominal es una manera simple de estimar el compartimiento adiposo abdominal. Sin embargo, no es capaz de diferenciar entre sus componentes subcutáneo e intraabdominal. Por este motivo, se han usado numerosos métodos radiológicos para la cuantificación de la grasa omental y mesentérica, pero cada uno de ellos tiene desventajas que dificultan su uso en la práctica clínica. La resonancia nuclear magnética (RNM) tiene un acceso limitado debido a su alto costo; la tomografía axial computarizada (TAC) es cara y conlleva una exposición a radiaciones ionizantes; el ultrasonido (US) es operador dependiente y no ha sido totalmente estandarizado para este tipo de mediciones ${ }^{6,7}$. A pesar de esto, el US es barato, seguro y accesible. Además, esta metodología ha mostrado buenas correlaciones con mediciones tomográficas de adiposidad intraabdominal, por lo que ha sido utilizada por diversos grupos de investigación ${ }^{7-10}$.

La limitada información que existe sobre estimaciones de adiposidad por US en la población pediátrica, sumado a la necesidad de contar con un examen simple que identifique sujetos en mayor riesgo cardiovascular, hacen que este tipo de estudios sea de particular interés. En este contexto, el objetivo de este estudio fue evaluar la asociación de las mediciones radiológicas de adiposidad intraabdominal con las comorbilidades propias de la obesidad en escolares obesos.

\section{Pacientes y Métodos}

Pacientes: El estudio de corte transversal, descriptivo. Se seleccionaron treinta pacientes obesos (índice de masa corporal (IMC) $\geq$ percentil 95 para sexo y edad) del Policlínico de Nutrición del Hospital Clínico San Borja Arriarán (HCSBA), Santiago, Chile ${ }^{11}$. Fueron incluidos pacientes que tuvieran 6 años o más, en estadío I de desarrollo puberal según Tanner, que no hubieran iniciado el tratamiento de obesidad ${ }^{12}$. Se excluyeron los pacientes con enfermedad crónica o aguda o los que usaran algún medicamento. El protocolo fue aprobado por el Comité de Ética del HCSBA y del Instituto de Nutrición y Tecnología de los Alimentos (INTA). En cada caso el tutor legal del niño otorgó un consentimiento informado.
Protocolo: Los pacientes fueron recibidos a primera hora de la mañana, tras 10 horas de ayuno, en las dependencias del HCSBA. Un único médico examinó a todos los pacientes. Se descartó la presencia de enfermedad mediante historia clínica y examen físico. Se cuantificó peso y estatura en balanza y estadiómetro Detecto ${ }^{\circledR}$, con los niños descalzos y en ropa interior. La medición de la circunferencia de cintura fue realizada a nivel del ombligo, tras espiración normal, con huincha métrica flexible. La presión arterial fue evaluada a través de un esfigmomanómetro de mercurio. Se tomaron muestras de sangre para el análisis de glicemia, insulinemia y perfil lipídico (Diagnostic System Laboratory $\left.{ }^{\circledR} \mathrm{Wb}, \mathrm{Tx}\right)$. Durante la misma mañana, aún en ayunas, se realizaron los exámenes de US y TAC en el Departamento de Radiología del HCSBA.

El puntaje z de la talla y el IMC para sexo y edad, así como la distribución percentilar, fueron analizados según las referencias del Centro de Control de Enfermedades ${ }^{11}$. En el caso de la circunferencia de cintura, los percentiles específicos para sexo y edad fueron evaluados usando los estándares publicados por Fernandez y cols ${ }^{13}$. La estimación del grado de sensibilidad insulínica fue realizada a través del cálculo del valor de HOMA, definiendo la presencia de insulino-resistencia (IR) según las referencias nacionales para niños prepuberales: $10 \mathrm{mU} / \mathrm{mL}$ para insulina y 2,1 para $\mathrm{HOMA}^{14,15}$. Para la presión arterial se utilizaron las referencias de la Sociedad Americana de Pediatría, mientras que los lípidos séricos fueron clasificados según los criterios americanos ${ }^{16,17}$. Por último, se estudió la presencia de síndrome metabólico utilizando los criterios pediátricos que Cook y cols adaptaron desde los definidos previamente para adultos ${ }^{18,19}$.

Técnicas de imágenes: El mismo operador entrenado realizó todas las mediciones ultrasonográficas de tejido adiposo subcutáneo e intraabdominal, según lo descrito previamente ${ }^{7}$. Brevemente, el segmento adiposo subcutáneo e intraabdominal fue medido con el transductor ubicado $1 \mathrm{~cm}$ cefálico al ombligo, en la línea xifo-umbilical, ejerciendo sobre la pared abdominal la mínima presión necesaria para permitir la visualización de la vértebra. Las mediciones de los segmentos fueron realizadas desde imágenes congeladas en la pantalla (equipo de ultrasonido ATLHDI 3000, con transductor 
sectorial electrónico 3,5 MHz), usando calibradores electrónicos posicionados en las interfaces piel-grasa o grasa-músculo y en la cara ventral de la vértebra. Estas mediciones fueron realizadas en triplicado para cada individuo. De esta manera, para cada sujeto se obtuvo el promedio de un segmento piel-músculo (correspondiente al segmento adiposo subcutáneo) y el promedio del segmento piel-vértebra (que permitió el cálculo del segmento adiposo intraabdominal, al restarle el segmento adiposo subcutáneo). Además, por medio de TAC (General Electric, model Lightspeed, Multislice), se realizó un único corte transversal de $10 \mathrm{~mm}$ a nivel del disco intervertebral L4-L5 ${ }^{6}$. Las áreas de tejido adiposo (intraabdominal y subcutáneo) fueron medidas usando el programa de contorno disponible en el tomógrafo, según lo descrito previamente ${ }^{20}$.

Estadística: Las variables numéricas continuas con distribución normal (según prueba de Shapiro-Wilk) fueron presentadas por su promedio $y$ desviación estándar (DE) y fueron analizadas por estadística paramétrica (prueba t y correlación de Pearson). Las variables que no tenían distribución normal fueron presentadas por su mediana y rango intercuartil, siendo analizadas por estadística no paramétrica (comparación de Mann Whitney y correlación de Spearman). Los análisis de regresión múltiple se realizaron con variables dependientes normales o normalizadas (transformadas a su logaritmo natural). Finalmente se realizaron análisis de curvas ROC (Receiver Operating Characteristic) para determinar el valor del segmento adiposo intraabdominal que identifica la presencia de alteraciones metabólicas relacionadas con obesidad. Los análisis fueron realizados en el programa estadístico SPSS. El tamaño muestral fue calculado según el coeficiente de correlación entre mediciones de tejido adiposo intraabdominal e insulinemia basal reportado por Guldiken y cols $(\mathrm{r}=0,548)$, utilizando un error alfa de $5 \%$ y un poder de $90 \%{ }^{21}$.

\section{Resultados}

La Tabla 1 muestra las características generales de los 30 pacientes que participaron en el estudio. Una importante proporción de ellos presentó alteraciones propias de la obesidad: $87 \%$ tenía una circunferencia abdominal $\geq$ al percentil 90 . El 27\% tuvo presión arterial elevada según sexo y edad (de ellos, el 100\% tuvo la presión arterial diastólica $(\mathrm{PAD})$ elevada, mientras que sólo un tercio presentó una presión arterial sistólica (PAS) alterada). Ningún sujeto presentó glicemia alterada de ayunas ( $\geq 100 \mathrm{mg} / \mathrm{dL})$, sin embargo, la insulinemia de ayunas estuvo elevada en $67 \%$ de los niños, con $47 \%$ catalogado como IR según HOMA. Las alteraciones lipídicas fueron: $47 \%$ de colesterol total elevado ( $\geq 170 \mathrm{mg} / \mathrm{dL}$ ); $23 \%$ de colesterol LDL elevado ( $\geq 110 \mathrm{mg} / \mathrm{dL})$; colesterol HDL alterado ( $\leq 40 \mathrm{mg} / \mathrm{dL}$ ) en $27 \%$ de la muestra. Los valores de triglicéridos (TAG) fueron iguales o mayores a $110 \mathrm{mg} / \mathrm{dL}$ en $47 \%$; $30 \%$ de los niños tuvo 3 o más criterios positivos de síndrome metabólico.

Las asociaciones entre las mediciones de tejido adiposo y las variables metabólicas se muestran en la Tabla 2. En ella se aprecia que las correlaciones obtenidas para el segmento adiposo intraabdominal medido por US son mejores que las obtenidas para antropometría. Ya que la muestra contaba con sujetos de diferente sexo, edad y estatura, quisimos evaluar el efecto que estas variables (junto con la superficie corporal) tenían sobre los valores del segmento intraabdominal medido, a través de un modelo de regresión múltiple. El análisis mostró que sólo la superficie corporal se asoció de forma significativa al segmento intraabdominal $\left(\mathrm{R}^{2}=0,25 ; \mathrm{p}<0,01\right)$. Al analizar nuevamente las correlaciones entre las variables metabólicas y segmento ultrasonográfico, ajustando por superficie y estatura, las asociaciones mantuvieron su significancia estadística.

Se realizó análisis por curvas ROC con la finalidad de obtener un punto de corte a partir del cual el segmento adiposo intraabdominal fuera capaz de discriminar a los sujetos que presentan complicaciones metabólicas. Un segmento ultrasonográfico superior a $45 \mathrm{~mm}$ fue el punto de corte que mejor determinó la presencia de factores de riesgo cardiovascular. En la Tabla 3 se señalan las sensibilidades y especificidades de esta medición para las diferentes condiciones asociadas a obesidad. Además se presentan las áreas bajo la curva obtenidas para el segmento adiposo intra-abdominal y la circunferencia abdominal.

Se agruparon los sujetos según las categorías determinadas por el punto de corte propuesto, 
Mediciones de adiposidad intraabdominal por ultrasonido en niños obesos - M. Reyes et al

lo que se visualiza en la Tabla 4. Este criterio fue capaz de discriminar dos grupos muy diferentes (en términos de variables metabólicas) de niños obesos.
Por último, la Figura 1 muestra las correlaciones obtenidas entre el segmento adiposo intraabdominal medido por US y el área de tejido adiposo intraabdominal obtenido por TAC.

Tabla 1. Características generales de la muestra agrupada por sexo

\begin{tabular}{|c|c|c|c|c|}
\hline Variables & $\begin{array}{l}\text { Hombres } \\
(n=15)\end{array}$ & & $\begin{array}{l}\text { Mujeres } \\
(n=15)\end{array}$ & Valor $\mathbf{p}$ \\
\hline Edad [años]* & $( \pm 1,7)$ & 8,5 & $( \pm 0,99)$ & $<0,05$ \\
\hline Estatura $[\mathrm{cm}]^{*}$ & $139,6( \pm 10,2)$ & 132,1 & $( \pm 8,4)$ & $<0,05$ \\
\hline Superficie $\left[\mathrm{m}^{2}\right]^{*}$ & $1,37 \quad( \pm 0,22)$ & 1,25 & $( \pm 0,17)$ & NS \\
\hline $\mathrm{zIMC**}$ & $(2,4-35)$ & 2,9 & $(2,2-4,2)$ & NS \\
\hline Circunferencia abdominal [cm]* & $( \pm 10)$ & 82 & $( \pm 9,3)$ & NS \\
\hline PAS $[\mathrm{mm} \mathrm{Hg}]^{*}$ & $104,5( \pm 11,6)$ & 97,3 & $( \pm 9,6)$ & NS \\
\hline $\operatorname{PAD}[\mathrm{mm} \mathrm{Hg}]^{*}$ & $69,9 \quad( \pm 12,4)$ & 63,1 & $( \pm 8,3)$ & NS \\
\hline Glicemia [mg/dL]* & $76,2 \quad( \pm 8,9)$ & 76,3 & $( \pm 4,7)$ & NS \\
\hline Insulinemia $[\mu \mathrm{U} / \mathrm{dL}]^{* *}$ & $(6,4-11,4)$ & 12,1 & $(10,6-25,9)$ & $<0,01$ \\
\hline $\mathrm{HOMA}^{* *}$ & $1,68 \quad(1,25-2,13)$ & 2,31 & $(1,9-4,97)$ & $<0,01$ \\
\hline Insulino-resistencia (+) & $33,3 \%$ & $60 \%$ & & NS \\
\hline Colesterol total [mg/dL] ${ }^{*}$ & $165,4( \pm 31,2)$ & 155,7 & $( \pm 33,9)$ & NS \\
\hline Colesterol LDL [mg/dL]* & $96,7 \quad( \pm 25,1)$ & 77,3 & $( \pm 21,1)$ & $<0,05$ \\
\hline Colesterol HDL [mg/dL]* & $45,6 \quad( \pm 5,6)$ & 43,7 & $( \pm 12,3)$ & NS \\
\hline Triglicéridos $[\mathrm{mg} / \mathrm{dL}]^{* *}$ & $(71-138,5)$ & 72 & $(61-239)$ & NS \\
\hline Criterios (+) sd. metabólico** & $2(1-3)$ & 1 & $(1-3)$ & NS \\
\hline Sd. metabólico (+) & $33,3 \%$ & $26,7 \%$ & & NS \\
\hline Área adiposa intra-abdominal (TAC) $\left[\mathrm{mm}^{2}\right]^{* *}$ & $2.909(1.815-3.701)$ & 3.418 & $(1.974-4.111)$ & NS \\
\hline Segmento adiposo intra-abdominal (US) [mm] ${ }^{*}$ & $47(11)$ & 49 & $(15)$ & NS \\
\hline
\end{tabular}

*Variables numéricas que tienen distribución normal, representadas por su promedio $( \pm \mathrm{DE})$, comparadas por prueba $t$. **Variables numéricas continuas que no distribuyen normal o variables numéricas categóricas, representadas por mediana (rango intercuartil), comparadas por Mann Whitney. zIMC: puntaje z para índice de masa corporal; PAS: presión arterial sistólica; PAD: presión arterial diastólica; TAC: tomografía axial computarizada; US: ultrasonido; Sd, metabólico definido utilizando criterios de Cook (19).

Tabla 2. Asociaciones entre estimaciones de adiposidad y variables metabólicas

\begin{tabular}{|lccc|}
\hline Variables & zIMC & $\begin{array}{c}\text { Circunferencia } \\
\text { abdominal }\end{array}$ & $\begin{array}{c}\text { Segmento adiposo } \\
\text { intra-abdominal (US) }\end{array}$ \\
\hline Insulina & 0,27 & 0,34 & $0,47^{* *}$ \\
\hline HOMA & 0,28 & 0,32 & $0,47^{* *}$ \\
\hline Triglicéridos & 0,2 & 0,23 & $0,46^{*}$ \\
Criterios (+) sd. metabólico & 0,34 & $0,6^{* *}$ & $0,66^{* *}$ \\
\hline
\end{tabular}

Análisis estadístico por correlación de Spearman, * representa valor de $\mathrm{p}<0,05$. **representa valor de $\mathrm{p}<0,01$. zIMC: puntaje z para índice de masa corporal. US: ultrasonido. Sd, metabólico definido utilizando criterios de Cook (19). 
Mediciones de adiposidad intraabdominal por ultrasonido en niños obesos - M. Reyes et al

Tabla 3. Sensibilidad y especificidad con que se identifica a pacientes con variables metabólicas alteradas utilizando el punto de corte propuesto para segmento adiposo intra-abdominal (> $45 \mathrm{~mm}$ )

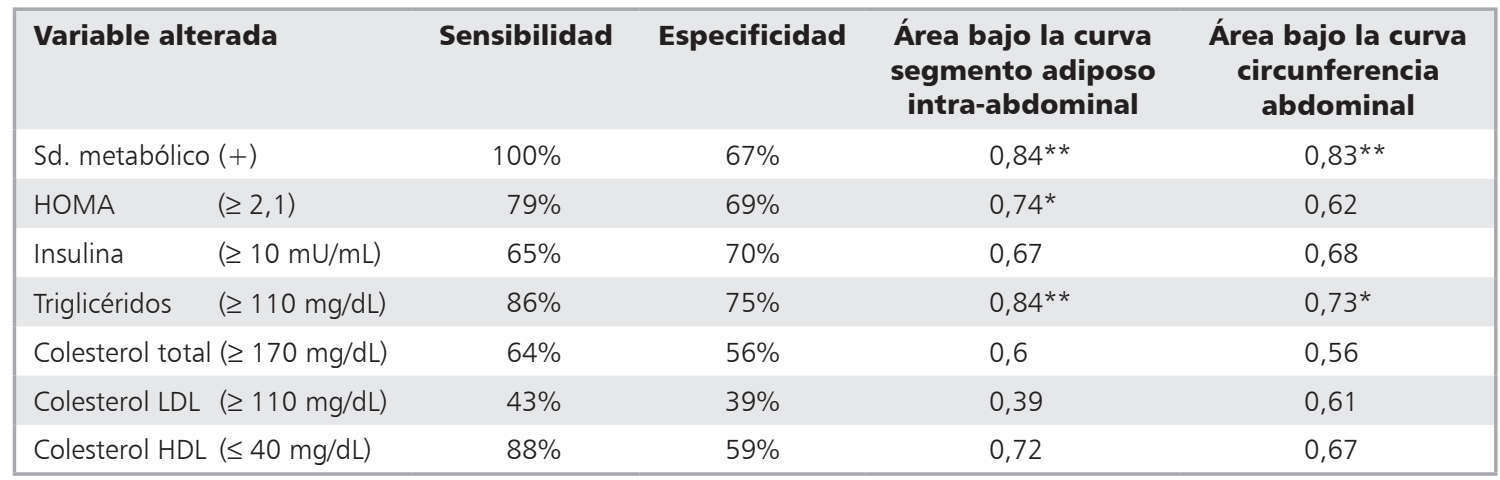

Sd, metabólico definido utilizando criterios de Cook (19). Análisis estadístico por curvas ROC, * representa valor de $p<0,05$. **representa valor de $\mathrm{p}<0,01 . \mathrm{H}_{0}$ : área bajo la curva $=0,5$.

Tabla 4. Características generales de la muestra categorizada según segmento adiposo intra-abdominal (US)

\begin{tabular}{|c|c|c|c|}
\hline \multirow[t]{2}{*}{ Variables } & \multicolumn{2}{|c|}{ Segm. Adiposo Intra-abdominal (US) } & \multirow[t]{2}{*}{ Valor $\mathbf{p}$} \\
\hline & $\leq 45 \mathrm{~mm}(\mathrm{n}=14)$ & $>45 \mathrm{~mm}(\mathrm{n}=16)$ & \\
\hline Sexo masculino & $43 \%$ & $56 \%$ & NS \\
\hline Edad [años]* & $8,7 \quad( \pm 1)$ & $9,6 \quad( \pm 1,8)$ & NS \\
\hline Estatura $[\mathrm{cm}]^{*}$ & $132,6( \pm 7,1)$ & $138,8( \pm 11,3)$ & NS \\
\hline Superficie $\left[m^{2}\right]^{*}$ & $1,22 \quad( \pm 0,15)$ & $1,4 \quad( \pm 0,2)$ & $<0,05$ \\
\hline $\mathrm{zIMC}^{* *}$ & $2,6 \quad(2,3-3,2)$ & $3,7 \quad(2,6-4,4)$ & NS \\
\hline Circunf. abdominal $[\mathrm{cm}]^{*}$ & $78 \quad( \pm 8,6)$ & $87,4 \quad( \pm 8,3)$ & $<0,01$ \\
\hline PAS $[\mathrm{mm} \mathrm{Hg}]^{*}$ & $98,3 \quad( \pm 10,9)$ & $103,3( \pm 11,1)$ & NS \\
\hline $\mathrm{PAD}[\mathrm{mm} \mathrm{Hg}]^{*}$ & $62,9 \quad( \pm 11,1)$ & $70 \quad( \pm 10,1)$ & NS \\
\hline Glicemia [mg/dL]* & $76,4 \quad( \pm 5,4)$ & $76,1 \quad( \pm 8,3)$ & NS \\
\hline Insulinemia $[\mathrm{mU} / \mathrm{dL}]^{* *}$ & $10,1 \quad(6,3-10,9)$ & $13,5 \quad(10,3-19,6)$ & $<0,05$ \\
\hline $\mathrm{HOMA}^{* *}$ & $1,82 \quad(1,17-2,02)$ & $2,68 \quad(1,81-3,77)$ & $<0,05$ \\
\hline Insulino-resistencia (+) & $21 \%$ & $69 \%$ & $<0,05$ \\
\hline Colesterol total $[\mathrm{mg} / \mathrm{dL}]^{*}$ & $151,7( \pm 26)$ & $168,3( \pm 36,1)$ & NS \\
\hline Colesterol LDL [mg/dL]* & $86,6 \quad( \pm 24)$ & $87,3 \quad( \pm 26,3)$ & NS \\
\hline Colesterol HDL [mg/dL]* & $48,4 \quad( \pm 8,2)$ & $41,3 \quad( \pm 9,4)$ & $<0,05$ \\
\hline Triglicéridos $[\mathrm{mg} / \mathrm{dL}]^{* *}$ & $69 \quad(58,8-76)$ & $149 \quad(109,5-224,5)$ & $<0,01$ \\
\hline Criterios (+) sd. metabólico** & $1 \quad(1-1)$ & $3(2-3)$ & $<0,01$ \\
\hline Sd. metabólico (+) & $0 \%$ & $56 \%$ & $<0,01$ \\
\hline Segmento adiposo intra-abdominal (US) [mm]* & $37,81( \pm 7,88)$ & $56,85( \pm 9,89)$ & $<0,01$ \\
\hline
\end{tabular}

*Variables numéricas que tienen distribución normal, representadas por su promedio $( \pm \mathrm{DE})$, comparadas por prueba t. **Variables numéricas continuas que no tienen distribución normal o variables numéricas categóricas, representadas por mediana (rango intercuartil), comparadas por Mann Whitney. zIMC: puntaje z para índice de masa corporal. PAS: presión arterial sistólica. PAD: presión arterial diastólica. US: ultrasonido. Sd, metabólico definido utilizando criterios de Cook (19). 


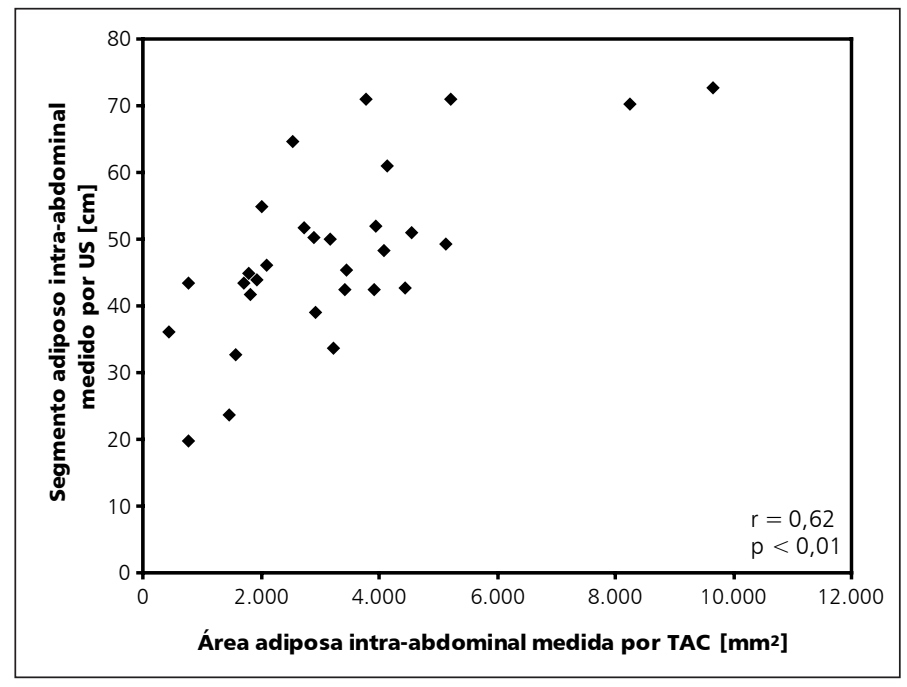

Figura 1. Correlación entre estimaciones radiológicas de depósito adiposo intra-abdominal: área (medida por TAC) y segmento (medido por US). Análisis Estadístico: correlación de Spearman. TAC: tomografía axial computarizada. US: ultrasonido.

\section{Discusión}

La medición del segmento adiposo intraperitoneal por US estuvo correlacionada de forma significativa con la intensidad de los factores de riesgo cardiovascular en escolares obesos. Esta medición mostró, además, tener una buena asociación con las mediciones de área intraabdominal medida por TAC.

Un corte transversal del abdomen, por TAC o RNM, es el estándar de oro para la estimación del compartimiento adiposo intraabdominal. La primera implica exposición a rayos $\mathrm{X}$ y la segunda tiene un alto costo asociado. Intentamos, por tanto, realizar mediciones ecográficas de este compartimiento, usando la metodología descrita por Armellini y cols ${ }^{7}$. Esta técnica demostró ser precisa, con una variación inter-observador de $4,1 \%{ }^{22}$. Además se obtuvieron correlaciones entre técnica ecográfica y tomográfica similares a las descritas previamente por otros autores ${ }^{7,8}$.

Algunas variables metabólicas de importancia estuvieron significativamente asociadas al segmento medido por US, lo que se mantuvo tras ajustar por superficie corporal y estatura. No encontramos en la literatura otros estudios con mediciones similares en población pediátrica para comparar nuestros resultados. Guldiken y cols, estudiaron adultos con diagnóstico de síndrome metabólico, encontrando asociaciones similares entre segmento adiposo, insulina, TAG y presión arterial $^{21}$. Guimaraes y cols, estudiaron pacientes infectados con el virus de inmunodeficiencia humana, encontrando que un segmento adiposo intraabdominal mayor de $70 \mathrm{~mm}$ se asociaba a un perfil lipídico aterogénico, resistencia insulínica y presión arterial elevada ${ }^{9}$. No logramos identificar asociaciones como las que ellos reportan para presión arterial. Estas diferencias pudieran deberse a que en nuestro grupo etario las patologías asociadas a obesidad están recién comenzando, por lo que aún no se manifestarían alteraciones de la presión arterial, que serían más tardías. Tamura y cols, han asociado mediciones de tejido adiposo intraabdominal obtenidas por US con variables metabólicas de niños obesos ${ }^{10}$. Las asociaciones que ellos presentan son mejores a las que nosotros reportamos, pero los resultados no son del todo comparables debido a que su muestra incluía pacientes adolescentes, etapa de IR, lo que podría aumentar la fuerza de las asociaciones. Por otra parte, la técnica que ellos utilizaban se basaba en la medición de la grasa pre-peritoneal ${ }^{23}$.

El análisis por curvas ROC mostró que el segmento adiposo intraabdominal presentaba un área bajo la curva significativa estadísticamente $y$, por lo tanto, sensibilidades y especificidades adecuadas para la identificación de la mayoría de las variables metabólicas alteradas. Estos valores fueron discretamente mejores que los encontrados para circunferencia abdominal. Las sensibilidades y especificidades obtenidas para el punto de corte propuesto $(45 \mathrm{~mm})$ debieran mejorar en futuros estudios enfocados en la búsqueda de criterios es- 
pecíficos por edad. De hecho el valor de segmento intraabdominal discriminante de riesgo aumenta en adolescentes y adultos ${ }^{9,24}$.

Aun cuando la evidencia actual no nos permite determinar si el tejido adiposo intraabdominal tiene una relación causal con la presencia de factores de riesgo cardiovascular o si es tan solo un marcador de este tipo de alteraciones, es claro que el tamaño del depósito visceral está relacionado con la presencia de alteraciones metabólicas. La disponibilidad de métodos para su cuantificación es un aporte en la identificación de sujetos en mayor riesgo.

En la actualidad, la obesidad infantil representa un problema epidemiológico importante y no poseemos ni los fondos ni la organización necesaria para tratar a todos los niños en esta condición ${ }^{25}$. Es por esto que se necesita el desarrollo de métodos que permitan identificar aquellos sujetos en mayor riesgo biológico, los que debieran ser sometidos a un estudio y manejo más enérgico. De esta manera podremos optimizar los recursos disponibles.

Nuestra propuesta no supone que cada niño obeso debiera ser sometido a ecografía abdominal aislada para la evaluación de su estado metabólico. Sin embargo, esta medición podría sumarse a otras evaluaciones de US que están siendo utilizadas en niños obesos, como son la evaluación de hígado graso, la medición del grosor de íntima-media carotídeo y el estudio de ovario poliquístico. De esta forma podría ser un aporte como tamizaje secundario de subgrupos de niños obesos que requieren un seguimiento más acucioso y mantenido en el tiempo. La identificación de un paciente en riesgo, induciría al médico tratante a un estudio más acabado y derivación oportuna para su manejo.

En conclusión, se encontraron asociaciones significativas entre segmento adiposo intraabdominal estimado por US y la presencia de factores de riesgo cardiovascular, las que permitieron discriminar la presencia de alteraciones en los parámetros metabólicos entre los niños obesos.

Agradecimientos: Agradecemos a la E.U. Alejandra Âvila y el Dr. Germán Iñiguez por la toma de muestras sanguíneas y las mediciones de insulina.

\section{Referencias}

1. Daniels SR. The consequences of childhood overweight and obesity. Future Child 2006; 16: 47-67.
2. Bjorntorp P. Abdominal fat distribution and disease: an overview of epidemiological data. Ann Med 1992; 24: 15-8.

3. Goran M, Gower B. Relation between visceral fat and disease risk in children and adolescents. Am J Clin Nutr 1999; 70: S149-56.

4. Lee S, Bacha F, Arslanian SA. Waist circumference, blood pressure, and lipid components of the metabolic syndrome. J Pediatr 2006; 149: 809-16.

5. Frayn K. Visceral fat and insulin resistance - causative or correlative? Br J Nutr 2000; 83: S71-7.

6. Wajchenberg BL. Subcutaneous and visceral adipose tissue: their relation to the metabolic syndrome. Endocr Rev 2000; 21: 697-38.

7. Armellini F, Zamboni M, Rigo L, Tudesco T, BergamoAndreis IA, et al. The contribution of sonography to the measurement of intra-abdominal fat. J Clin Ultraosound 1990; 18: 563-7.

8. Ferrozzi F, Zuccoli G, Tognini G, Castriota-Scanerberg A, Bacchini E, Bernasconi S, et al. An assessment of abdominal fatty tissue distribution in obese children. A comparison between echography and computed tomography]. Radiol Med (Torino) 1999; 98: 490-4.

9. Guimaraes M, De Oliveira Junior A, Penido M, Queiroz L, Goulart E, et al. Ultrasonographic measurement of intra-abdominal fat thickness in HIV-infected patients treated or not with antiretroviral drugs and its correlation to lipid and glycemic profiles. Ann Nutr Metab 2007; 51: 35-41.

10. Tamura A, Mori T, Hara Y, Komiyama A. Preperitoneal fat thickness in childhood obesity: association with serum insulin concentration. Pediatr Int 2000; 42: 155-9.

11. Centers for Disease Control, National Center for Health Statistics. Individual growth charts. Disponible en: http://www.cdc.gov/growthcharts [Consultado el 30 de enero de 2009].

12. Tanner JM. Growth at adolescence with a general consideration of the effects of hereditary and environmental factors upon growth and maturation from birth to maturity, 2 ed. Oxford: Blackwell, Scientific Publications; 1962.

13. Fernández J, Redden D, Pietrobelli A, Allison D. Waist circumference percentiles in nationally representative samples of African-American, European-American, and Mexican-American children and adolescents. J Pediatr 2004; 145: 439-44.

14. Matthews DR, Hosker JP, Rudenski AS, Naylor BA, Treacher DF, Turner RC. Homeostasis model assessment: insulin resistance and beta-cell function from fasting plasma glucose and insulin concentrations in man. Diabetología 1985; 28: 412-9. 
15. Burrows R, Leiva L, Burgueño M, Maggi A, Giadrosic V, Díaz E, et al. Sensibilidad insulínica en niños de 6 a 15 años: asociación con estado nutricional y pubertad. Rev Med Chile 2006; 134: 1417-26.

16. American Academy of Pediatrics. Cholesterol in childhood. Pediatrics 1998; 101: 141-7.

17. National High Blood Pressure Education Program Working Group on High Blood Pressure in Children and Adolescents. The fourth on the diagnosis, evaluation and treatment of high blood pressure in children and adolescents. Pediatrics 2004; 114: 555-76.

18. Cook S, Weitzman M, Auinger P, Nguyen M, Dietz WH. Prevalence of a metabolic syndrome phenotype in adolescents. Finding from the Third National Health and Nutrition Examination Survey, 1988-1994. Arch Pediatr Adolesc Med 2003; 157: 821-7.

19. National Cholesterol Education Program. Detection, evaluation and treatment of high blood cholesterol in adults (Adult Treatment Panel III): Full Report. Bethesda, Md: National Institute of Health; 2001. NIH Publication No. 01-3670.

20. Shen W, Wang Z, Punyanita M, Lei J, Sinav A, Kral JG, et al. Adipose tissue quantification by imaging methods: a proposed classification. Obes Res 2003; 11: 5-16.

21. Guldiken S, Tuncbilek N, Okten OO, Arikan E, Tugrul A. Visceral fat thickness determined using ultrasonography is associated with anthropometric and clinical parameters of metabolic syndrome. Int J Clin Pract 2006; 60: 1576-81.

22. Espinoza A, Reyes M, Moraga F, Rebollo MJ, Mericq V, Castillo C. Mediciones ecográficas y tomografía computada de adiposidad y alteraciones metabólicas asociadas a obesidad en niños. Rev Chil Radiol 2006; 12: 172-6.

23. Suzuki R, Watanabe S, Hirai Y, Akiyama K, Nishide T, Matsushima $Y$, et al. Abdominal wall fat index, estimated by ultrasonography, for assessment of the ratio of visceral fat to subcutaneous fat in the abdomen. Am J Med 1993; 95: 309-14.

24. Neri D, Espinoza A, Bravo A, Rebollo MJ, Moraga F, Mericq V, Castillo-Durán C. Adiposidad visceral y su asociación con lípidos séricos e insulinemia en adolescentes obesas. Rev Med Chil 2007; 135 (3): 294-300.

25. Reilly JJ. Tackling the obesity epidemic: new approaches. Arch Dis Child 2006; 91: 724-6. 